\title{
The origin of galactic metal-rich stellar halo components with highly eccentric orbits
}

\author{
Azadeh Fattahi $^{1 \star}$, Vasily Belokurov ${ }^{2,3}$, Alis J. Deason ${ }^{1}$, Carlos S. Frenk ${ }^{1}$, \\ Facundo A. Gómez ${ }^{4,5}$, Robert J. J. Grand ${ }^{6,7,8}$, Federico Marinacci ${ }^{9}$, \\ Rüdiger Pakmor ${ }^{8}$, Volker Springel ${ }^{8}$ \\ ${ }^{1}$ Institute for Computational Cosmology, Department of Physics, University of Durham, South Road, Durham DH1 3LE, UK \\ ${ }^{2}$ Institute of Astronomy, Madingley Road, Cambridge CB3 OHA \\ ${ }^{3}$ Center for Computational Astrophysics, Flatiron Institute, 162 5th Avenue, New York, NY 10010, USA \\ ${ }^{4}$ Instituto de Investigación Multidisciplinar en Ciencia y Tecnología, Universidad de La Serena, Raúl Bitrán 1305, La Serena, Chile \\ ${ }^{5}$ Departamento de Física y Astronomía, Universidad de La Serena, Av. Juan Cisternas 1200 N, La Serena, Chile \\ ${ }^{6}$ Heidelberger Institut für Theoretische Studien, Schloß-Wolfsbrunnenweg 35, 69118 Heidelberg, Germany \\ 7 Zentrum für Astronomie der Universität Heidelberg, Astronomisches Recheninstitut, Mönchhofstr. 12-14, 69120 Heidelberg, Germany \\ ${ }^{8}$ Max-Planck-Institut für Astrophysik, Karl-Schwarzschild-Str. 1, D-85748, Garching, Germany \\ ${ }^{9}$ Harvard-Smithsonian Center for Astrophysics, 60 Garden St., Cambridge, MA 02138, USA
}

Accepted XXX. Received YYY; in original form ZZZ

\begin{abstract}
Using the astrometry from the ESA's Gaia mission, previous works have shown that the Milky Way stellar halo is dominated by metal-rich stars on highly eccentric orbits. To shed light on the nature of this prominent halo component, we have analysed 28 Galaxy analogues in the Auriga suite of cosmological hydrodynamics zoom-in simulations. Some three quarters of the Auriga galaxies contain prominent components with high radial velocity anisotropy, $\beta>0.6$. However, only in one third of the hosts do the high- $\beta$ stars contribute significantly to the accreted stellar halo overall, similar to what is observed in the Milky Way. For this particular subset we reveal the origin of the dominant stellar halo component with high metallicity, $[\mathrm{Fe} / \mathrm{H}] \sim-1$, and high orbital anisotropy, $\beta>0.8$, by tracing their stars back to the epoch of accretion. It appears that, typically, these stars come from a single dwarf galaxy with a stellar mass of order of $10^{9}-10^{10} \mathrm{M}_{\odot}$ that merged around $6-10 \mathrm{Gyr}$ ago, causing a sharp increase in the halo mass. Our study therefore establishes a firm link between the excess of radially anisotropic stellar debris in the halo and an ancient head-on collision between the young Milky Way and a massive dwarf galaxy.
\end{abstract}

Key words: galaxies: Milky Way, galaxies: dynamics

\section{INTRODUCTION}

The history of mass accretion onto the Milky Way can be constructed from the study of unrelaxed tidal debris in its stellar halo (e.g. De Lucia \& Helmi 2008; Johnston et al. 2008; Cooper et al. 2010). This works particularly well for the most recent events, such as the infall of the Sagittarius (Sgr) dwarf (see e.g. Ibata et al. 1994; Majewski et al. 2003). Yet, despite the fact that the Sgr stream has been mapped in glorious detail over the last decade or so (see Ibata et al. 2001; Majewski et al. 2003; Newberg et al. 2003; Belokurov et al. 2006; Yanny et al. 2009; Koposov et al. 2012), we have been remarkably slow to comprehend the damage this dwarf galaxy has been inflicting onto the Milky Way (see Purcell et al. 2011; Gómez et al. 2013; Laporte et al. 2018). Sgr is a striking example of how

* E-mail: azadeh.fattahi-savadjani@durham.ac.uk much destruction a massive satellite can bring to the fragile Milky Way's disc even on an orbit of moderate eccentricity (e.g. PriceWhelan et al. 2015; Hayes et al. 2018b; Bergemann et al. 2018; de Boer et al. 2018; Deason et al. 2018a).

Events in the distant past are normally less obvious, blurred by the debris' gradual relaxation in the host's gravitational potential and concealed behind the layers of the subsequent Galactic growth (e.g. Peñarrubia et al. 2006; Gómez \& Helmi 2010; Buist \& Helmi 2015; Erkal et al. 2016). Nonetheless, some episodes in the Milky Way's history are so cataclysmic that they are difficult to hide, even if they took place a long time ago.

It has been known for some time that the properties of the Milky Way stellar halo change drastically as a function of Galactocentric radius. This is revealed in the rapid variation of the stellar halo's kinematics and metallicity with radius (see e.g. Chiba \& Beers 2001; Carollo et al. 2007, 2010) as well as the halo's density 
profile (see Watkins et al. 2009; Deason et al. 2011; Sesar et al. 2011). Two possible explanations have been put forward, one invoking the presence of the so-called in-situ halo (Zolotov et al. 2009; McCarthy et al. 2012; Tissera et al. 2013; Cooper et al. 2015) and one appealing to an early accretion of a massive galaxy (Deason et al. 2013). While unambiguous evidence for an in-situ population is yet to be found (see e.g. Haywood et al. 2018), the hypothesis of a single accretion event dominating the nearby Galactic halo has been reinforced by multiple independent observations. These include the pattern of the stellar halo's alpha-abundances (see Venn et al. 2004; Tolstoy et al. 2009; Nissen \& Schuster 2010; de Boer et al. 2014; Hayes et al. 2018a), the ratio of Blue Straggler to Blue Horizontal Branch stars (Deason et al. 2015), the evolution in the mixture of RR Lyrae of different Oosterhoff types (Belokurov et al. 2018a) and the relative scarcity of the Galactic stellar halo substructure (Helmi et al. 2011; Xue et al. 2011; Lancaster et al. 2018a).

Most recently, with the help of the astrometry from the ESA's Gaia mission (Gaia Collaboration et al. 2016), the Milky Way halo has been demonstrated to contain a large number of metal-rich stars on extremely eccentric orbits (Belokurov et al. 2018b; Myeong et al. 2018b; Haywood et al. 2018; Helmi et al. 2018; Mackereth et al. 2018). Using the established mass-metallicity relationship (Kirby et al. 2013) as well as numerical simulations of Galaxy formation, Belokurov et al. (2018b) have argued that the observed chemistry and the drastic radial anisotropy of the halo stars (the "Gaia Sausage") are telltale signs of an ancient collision between the Milky Way and a massive dwarf. This conjecture builds on the insights into the stellar halo assembly presented in the work by Amorisco (2017), which for the first time posits a link between high radial anisotropy and the infall of a massive satellite. The idea of an ancient intergalactic crash has since received additional support with the discovery of a large number of globular clusters associated with this accretion event (see Myeong et al. 2018a; Kruijssen et al. 2018). Additionally, the enormous extent of the "Sausage" debris is now starting to become apparent with the studies of the stellar halo's anisotropy variation with radius (Bird et al. 2018; Lancaster et al. 2018b). The break in the stellar halo density has been revealed to correspond to the apocentric pile-up of the "Sausage" stars (see Deason et al. 2018b), while the large diffuse halo overdensities such as Hercules-Aquila and Virgo Clouds are now believed to be linked to the same accretion event (see Simion et al. 2018). While most of these studies rely on small subsamples of halo tracers with complete phase-space information, Wegg et al. (2018) and Iorio \& Belokurov (2018) have recently used large, all-sky catalogs of RR Lyrae with proper motions to decipher the kinematic properties of the halo. As these authors demonstrate, the bulk of the Galactic stellar halo between 5 and $30 \mathrm{kpc}$ - as traced by these old pulsating stars - is on strongly radial orbits, yielding estimates of $\beta \sim 0.9$.

The dominance of one massive accretion event is in good agreement with the general predictions of cosmological simulations. Several works have shown that the majority of stellar mass accreted onto Milky Way mass haloes is contributed by a small number of massive satellites (e.g. Bullock \& Johnston 2005; Cooper et al. 2010; Deason et al. 2016). The hypothesis of a small number of massive contributors is a direct consequence of the hierarchical dark matter model, and the extremely steep stellar-tohalo mass relation in the dwarf galaxy regime (e.g. Eke et al. 2004; Moster et al. 2013; Garrison-Kimmel et al. 2014; Brook et al. 2014; Sawala et al. 2016; Read et al. 2017; Jethwa et al. 2018; Fattahi et al. 2018; Simpson et al. 2018). Thus, despite the large numbers of low-mass dwarfs accreted by Milky Way-mass galaxies, the con- tribution of these low mass dwarfs to the total accreted stellar mass is small (see Deason et al. 2016).

Curiously, some of the first efforts to study the emergence of orbital patterns in simulated stellar haloes already saw trends similar to those recently discovered in the Milky Way (see e.g. Brook et al. 2003; Meza et al. 2005; Navarro et al. 2011). While those works were limited to a small number of hand-picked cases, here we strive to carry out a systematic analysis using a statistically significant sample of realistic simulations of Milky Way analogues. Monachesi et al. (2018) recently studied the global properties of stellar haloes in the Auriga simulations (Grand et al. 2017). Indeed, in agreement with earlier work, these authors find that the global properties of the stellar haloes, such as the average metallicity, total stellar halo mass, and density profile, are directly related to the small number of stellar halo progenitors.

In this work we use the Auriga simulations (Grand et al. 2017) to understand the relatively local stellar halo kinematics, within 20 kpc of the Galactic centre ${ }^{1}$, to compare with the recent Gaia results. Ours is not the first attempt to interpret the observed local stellar halo properties with the help of simulated Milky Way like haloes. Most recently, Mackereth et al. (2018) compared Gaia and APOGEE data with the orbital and chemical properties of the accreted halo stars in the EAGLE simulation suite (see Schaye et al. 2015; Crain et al. 2015). They come to the conclusion that to explain the measured $\alpha$ vs $[\mathrm{Fe} / \mathrm{H}]$ trend as a function of eccentricity, an early accretion of a massive dwarf galaxy is required. A similar conclusion is reached by Fernández-Alvar et al. (2018) who use the stellar haloes in hydrodynamical simulations of Aquarius MW-like haloes (Aquila, see e.g. Tissera et al. 2013; Scannapieco et al. 2009; Springel et al. 2008). The Auriga simulations used here provide a higher resolution compared to EAGLE, and it gives us access to a significantly larger sample size with richer physics compared to Aquila.

Our Paper is structured as follows. In Section 2 we describe the Gaia Data Release 2 observations of a "Sausage-like" component in the phase space of the stellar halo. We describe the Auriga simulations in Section 3 and make a comparison with the Gaia results. In particular, we show how these highly eccentric, metal-rich stellar halo components (referred to as the radially-biased components, hereafter) are also produced in the simulations. Finally, in Section 4 we summarise and discuss our main results.

\section{OBSERVATIONS}

As a point of reference we use the view of the Milky Way's stellar halo provided in Data Release 2 (GDR2, see Gaia Collaboration et al. 2018) of the Gaia mission (see Gaia Collaboration et al. 2016). Specifically, we use a sample of GDR2 stars with radial velocities measured by Gaia (see Cropper et al. 2018; Sartoretti et al. 2018; Katz et al. 2018). Out of $\sim 7 \times 10^{6}$ stars with available spectroscopic information, we select $\sim 12,000$ with good parallaxes, $\varpi / \sigma_{\varpi}>3$ and $2<|z| / \mathrm{kpc}<4,|b|>25^{\circ}$ and $[\mathrm{Fe} / \mathrm{H}] \leqslant-1$. The latter three of the above cuts, including that on the Gaia's template metallicity (see Katz et al. 2018, for details), are applied to minimise the Galactic disc contamination. We convert the celestial positions and parallaxes into Galactocentric Cartesian coordinates, assuming a Solar radius of $8 \mathrm{kpc}$ and a Sun's height above

1 Note that Monachesi et al. (2018) studied the stellar halo components at larger distances of $10-100 \mathrm{kpc}$. 

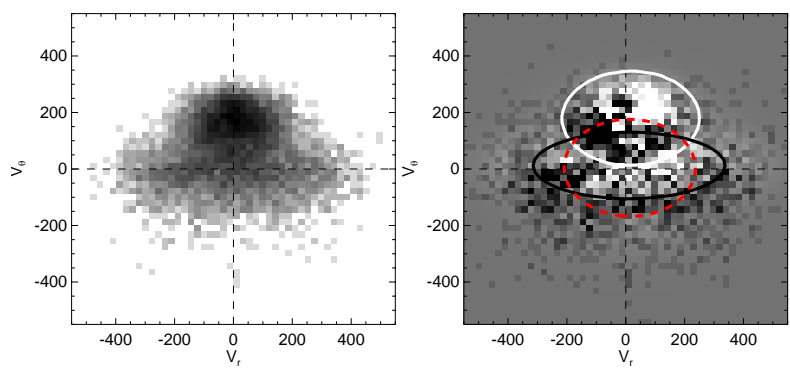

Figure 1. The "Gaia Sausage" as viewed with the GDR2 sample. Left: Logarithm of the stellar density in spherical $v_{r}, v_{\theta}$ coordinates, corresponding to the radial and azimuthal velocity components. Two prominent overdensities are visible, one corresponding to the (thick) disc with $\bar{v}_{\theta} \sim 170 \mathrm{~km} \mathrm{~s}^{-1}$ and one corresponding to the halo with no apparent rotation. Right: Residuals of the multi-variate three component Gaussian mixture model. Here excess (depletion) of the data with respect to the model is shown as a dark (light) region. Two thirds of the halo stars are in a radiallybiased ( $\beta \sim 0.86$ ) "Sausage" component indicated with a solid black line. The rest of the halo is in a much more isotropic component with $\beta \sim 0.4$ shown as dashed red line. Finally, at least half of this local GDR2 sample belongs to the disc, marked with a white solid line.

the Galactic plane of 0 . The proper motions and the line-of-sight velocities are translated into velocity components in spherical polars, using the Solar peculiar motion from Coşkunoğlu et al. (2011) and marginalising over the Local Standard of Rest (LSR) values assuming they are Gaussian distributed ${ }^{2}$ with a mean at $238 \mathrm{~km} \mathrm{~s}^{-1}$ and a standard deviation of $9 \mathrm{~km} \mathrm{~s}^{-1}$ (see Schönrich 2012). The uncertainties are propagated using Monte-Carlo sampling and the medians of the distributions for each object, as well as the scaled median absolute deviations, are used for the analysis below.

Fig. 1 shows the distribution of the median radial, $v_{r}$, and azimuthal, $v_{\theta}$, velocity components in the sample described above. Two populations are immediately apparent: the Galactic disc, with significant mean rotation and the non-rotating Galactic halo. We estimate the properties of each component by modelling the 3 D velocity distribution with a mixture of three multivariate Gaussians using the Extreme Deconvolution code (ED, see Bovy et al. 2011) which takes the measurement uncertainties into account. In this model, the Gaussians represent the disc and the two components of the halo, a radially-biased and a more isotropic one. The results of the ED fitting exercise are shown in the right panel of Fig. 1, where the residuals of data minus model are shown as a greyscale, with dark (white) regions corresponding to an excess (lack) of data points compared to the model prediction. Overlaid are the isodensity contours of the three Gaussians given by the best-fit model, with white solid representing the disc, black solid the radially-biased halo, and red dashed the second, more isotropic halo component.

As inferred from the ED modelling, $50 \%$ of our sample belongs to the (thick) disc with a mean rotation of $\bar{v}_{\theta} \sim 170 \mathrm{~km} \mathrm{~s}^{-1}$, $37 \%$ belong to the radially-biased halo population with $\beta \sim 0.86$ and $13 \%$ to the halo component with $\beta \sim 0.4$. More specifically, the radially-anisotropic "Sausage" component, whose radial, azimuthal and polar velocity dispersions are 177,61 and $70 \mathrm{~km} \mathrm{~s}^{-1}$ respectively, contains just over two thirds of the total halo population locally in good agreement with Belokurov et al. (2018b). In-

2 We are neglecting the asymmetric drift here, as we are interested in the $V_{\theta} \sim 0$ component where this effect is small. terestingly, according to this Gaussian mixture model, the radiallybiased component is centered on zero in all three velocity components, i.e. it exhibits no net rotation, in mild tension with some of the earlier results (e.g. Belokurov et al. 2018b; Necib et al. 2018), where a prograde rotation of $\sim 20 \mathrm{~km} \mathrm{~s}^{-1}$ was reported. The quality of the fit can be judged from the right panel of Fig. 1. Leaving aside the residuals at high $v_{\theta}$ associated with the disc, there is a dark/light pattern of under/over subtraction of the model from the data. The excess of observed stars with high $\left|v_{r}\right|$ velocities is consistent with previous modelling attempts. However, a less prominent but noticeable ridge of positive residuals exists at negative $v_{\theta}$. This implies that there may be a significant spray of retrograde debris possibly associated with the "Sausage" (see Myeong et al. 2018b; Helmi et al. 2018) not accounted for by our model. Judging by the shape of the data in the left panel of the figure as well as the residuals in the right, we conjecture that these residuals are likely due to the strongly non-Gaussian shape of the velocity distribution in the "Sausage" component. The complicated structure of the residuals advocates the use of more sophisticated models of the "Sausage" kinematics as those recently proposed in Necib et al. (2018) and Lancaster et al. (2018b). We, however, choose to use the relatively simple ED modeling here that can be applied to the simulations as well. This will enable us to make a comparison between the simulations and observations, and also between different simulated galaxies.

\section{AURIGA SIMULATIONS}

We use cosmological magneto-hydrodynamical (MHD) simulations of MW-like haloes from the Auriga project (Grand et al. 2017) to interprete the origin of the "Gaia Sausage" described in the previous section. The Auriga project includes a suite of $30 \mathrm{MW}$ size haloes simulated at high resolution, using the "zoom-in" technique (Frenk et al. 1996; Jenkins 2013) and the Tree-PM moving-mesh code Arepo (Springel 2010). The simulations have been performed at different levels of resolution. We use the standard resolution runs (level-4) in this study rather than the higher resolution ones (level3 ), as only eight haloes have been run at level-3. The mass resolution of the standard level- 4 runs is $5 \times 10^{4} \mathrm{M}_{\odot}$ and $3 \times 10^{5} \mathrm{M}_{\odot}$ per gas and dark matter particles, respectively. The selection of MW candidates, as well as the subgrid physics model, have been described in detail in Grand et al. (2017). Here we give a brief summary.

MW-like haloes were selected from the $100^{3} \mathrm{Mpc}^{3}$ dark matter-only periodic box of the EAGLE project (Schaye et al. 2015; Crain et al. 2015), based on their virial ${ }^{3}$ mass, $\sim 10^{12} \mathrm{M}_{\odot}$, and an isolation criterion. The subgrid physics model in the re-simulations includes a spatially uniform photoionizing UV background, primordial and metal line cooling, star formation, stellar evolution and supernovae feedback, supermassive black hole growth and feedback, and magnetic fields. A fixed set of chemical elements, including $\mathrm{Fe}$ and $\mathrm{H}$, have been consistently tracked within the simulations. Haloes and bound structures and substructures were identified using the FoF and SUBFIND algorithms (Davis et al. 1985; Springel et al. 2001), respectively. We refer to the central galaxy of the main halo in each Auriga simulation as a MW analogue. The cosmological parameters adopted for the simulations are those of 
Planck Collaboration XVI (Planck Collaboration et al. 2014): matter density of $\Omega_{m}=0.307$, baryonic density of $\Omega_{b}=0.048$, and a Hubble constant of $H_{0}=67.77 \mathrm{~km} \mathrm{~s}^{-1} \mathrm{Mpc}^{-1}$.

We use all $30 \mathrm{MW}$ analogues of the level-4 (standard) runs, except for Au-11 and Au-20. These two galaxies are undergoing a merger at the present time and this distorts the phase space distribution of star particles around the galactic discs.

The coordinate and velocity reference frame of the MW analogues are based on SUBFIND. The galactic discs are defined, and visually confirmed, using the net angular momentum of star particles in the inner $10 \mathrm{kpc}$. For our stellar samples, we keep star particles between $r=5-20 \mathrm{kpc}$ from the galaxy centre, with galactic latitude $|b|>15 \mathrm{deg}$, assuming a random azimuthal angle for the position of the Sun at $8 \mathrm{kpc}$ to roughly mimic the observational selections of Belokurov et al. (2018b) ${ }^{4}$. We only include stars bound to the MW analogues (not to the satellites) and shift the $[\mathrm{Fe} / \mathrm{H}]$ of star particles by a constant value of $\sim 0.5$ to roughly match the median and the [5th,95th] percentile of an equivalent sample in SDSS (Ahn et al. 2012). We adopt $-2.0,-0.83$, and -0.23 as the 5th, 50th, and 95th percentiles for SDSS stars, respectively.

We identify "accreted" star particles as those that were bound to galaxies other than the main progenitors of the MW analogues, at the snapshot following their formation time. According to this definition, stars that were formed out of the stripped gas from the satellites will be flagged as "in-situ" if they are bound to the MW analogues' progenitors right after their birth. We note that this identification method is dependent on the time resolution of the output snapshots. The temporal resolution is always better than $200 \mathrm{Myr}$ in Auriga level-4 simulations, and therefore, for our purposes, is not a major concern.

\subsection{The radially-biased stellar halo components in Auriga}

Following Belokurov et al. (2018b), we plot the distribution of stars in a $2 \mathrm{D}$ velocity space: azimuthal $\left(V_{\theta}\right)$ vs radial $\left(V_{\mathrm{r}}\right)$ in spherical coordinates, at different galactic height bins and metallicities for all our MW analogues. Examples of the velocity space in a grid of height and $[\mathrm{Fe} / \mathrm{H}]$ are presented in Appendix A. Here we focus our analysis on star particles at $9<|z| / \mathrm{kpc}<15$ and $-1<[\mathrm{Fe} / \mathrm{H}]<-0.7$. This galactic height range is larger than what was used in the MW analysis in Sec. 2. The reason behind our choice is that the differences between Auriga haloes are clearer at these heights and metallicities. At lower heights (but similar $[\mathrm{Fe} / \mathrm{H}])$, the distribution is dominated by stars in the galactic disc and at lower $[\mathrm{Fe} / \mathrm{H}]$ values, the radially-biased components become weaker. Our choice of sample selection criteria is reasonable since our goal is not to find an exact match to the MW's "Gaia Sausage" in the simulations, but rather more generally to investigate the origin of components with similar properties to those of the structure found in the MW.

The top row of Fig. 2 shows the velocity space of four example haloes (Au-5, Au-8, Au-28, and Au-30), for star particles at $9<|z| / \mathrm{kpc}<15$ and $-1<[\mathrm{Fe} / \mathrm{H}]<-0.7$. The rotating component with $V_{\theta} \sim 200 \mathrm{~km} \mathrm{~s}^{-1}$ in the top panels of Fig. 2 corresponds to the disc ${ }^{5}$. The halo components of these examples, which have much lower rotation (i.e. $V_{\theta} \sim 0$ ), appear very different. Au-5 has a prominent radially-biased component, similar to

\footnotetext{
4 We have checked that the results are not sensitive to these cuts.

5 This component is weak in Au-28 because the disc is more prominent only at lower heights.
}

the real MW in Fig. 1; while Au-8 lack such a component and are much more isotropic. These two galaxies illustrate the two ends of the spectrum of inner stellar halo kinematic features in the Auriga MW analogues.

The second row of Fig. 2 shows the velocity space of the accreted component only, with the same selection cuts as the top panels. We note that a large number of stars are removed from the top row when we select only accreted stars. For the particular spatial and metallicity selections used in this figure, the fraction of accreted stars is on average 30 per cent amongst 28 Auriga haloes. The fraction increases to 40 per cent if we relax the metallicity cut, mainly because the stellar halo is more metal poor. These numbers are overall consistent with the results of Monachesi et al. (2018). An exact comparison is not possible since they used different spatial and kinematic selections.

According to Fig. 2, the rotating (disc) component is, in most cases, not present in the accreted stars. A radially-biased component stands out in both $\mathrm{Au}-5$ and $\mathrm{Au}-28$, while the latter case is less prominent. $\mathrm{Au}-30$ and $\mathrm{Au}-8$, on the other hand, are isotropic. Moreover, Au- 8 has an accreted component in its disc. The existence of accreted (or "ex-situ") disc components is not surprising. Gómez et al. (2017) extensively discussed their origin in Auriga galaxies, and Rodriguez-Gomez et al. (2016) also report their existence in the Illustris simulations. Fig. 2 illustrates that the radially-biased component is more easily identified in the accreted stars. Moreover, the existence of an in-situ halo component in the MW is still under debate (see, e.g., Helmi et al. 2011; Bonaca et al. 2017; Deason et al. 2017; Haywood et al. 2018). We therefore include only accreted particles for the rest of our analysis, unless otherwise stated.

In order to quantify the kinematic components in Auriga we decompose the velocity space of stars, at different heights and metallicities, into multi-variate Gaussian components using the Extreme Deconvolution method, similar to Section 2. The details of the procedure are described in Appendix B. In summary, we first combine star particles for each MW analogue in various $[\mathrm{Fe} / \mathrm{H}]$ bins, and then decompose the velocity distribution at each galactic height into either two or three Gaussians, depending on the existence of a rotating (accreted) disc component. The velocity ellipsoids have 6 free parameters describing their centre and shape, at this stage. The three component fits include one for the disc and two for the halo-like stars; the two component fits typically include two halo-like ones. We visually inspect all 28 Auriga simulations to check that the fits are reasonable. For the second stage of the fitting, we fix the centre and shape of the ellipsoids from the previous step, and fit them to different $[\mathrm{Fe} / \mathrm{H}]$ bins at each height, letting the amplitude (i.e. contribution) of the components vary.

The bottom row of Fig. 2 shows the residuals of the fits to the accreted stars. The ellipses illustrate the components of the fits: a radially-biased halo component (black ellipse) and a more isotropic one (red dashed ellipse) for Au-5 and Au-28; two relatively isotropic halo components for Au-30 where one (black ellipse) is slightly more radial than the other (red ellipse); an isotropic halo component (black ellipse) and a disc component for Au-8 ${ }^{6}$. The black ellipse of Au-5, which is equivalent to the radially-biased component observed in Gaia, indicates $\beta \sim 0.9$ with a large contribution to the stellar distribution ( $\sim 90$ per cent). The highly eccentric component of $\mathrm{Au}-28$, however, contributes a much lower frac-

6 Au-8 is an exception where the two components of the fit include one for the disc and one for the halo. This is equivalent to a three component fit where the contribution of a radially-biased halo component is zero. 

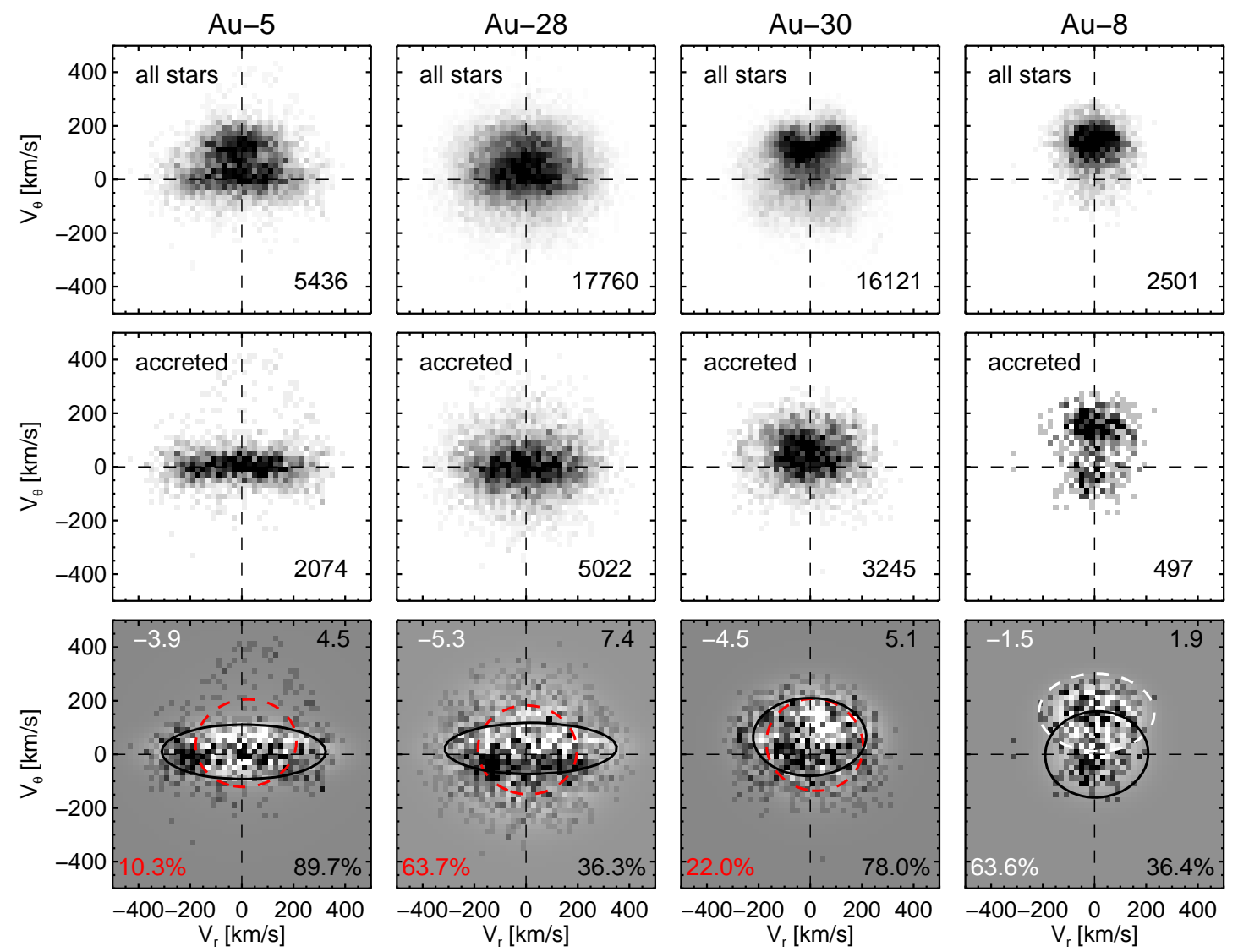

Figure 2. Distribution of star particles in velocity space for four Auriga halos (Au-5, Au-8, Au-28, Au-30) shown in different columns. The particles are located between $9-15 \mathrm{kpc}$ above and below the disc and have $-1<[\mathrm{Fe} / \mathrm{H}]<-0.7$. These examples illustrate the variety of kinematic features found in the inner stellar halo in Auriga galaxies. Au- 5 and Au- 28 have a highly radial population of stars, while Au- 8 and Au- 30 lack such a component. Top: Velocity space of all star particles in the given height and metallicity bin. The number of star particles in each panel is quoted in the legend. Middle: Similar to the top row but only for accreted stars. Bottom: The residuals of the fits to the velocity space of the accreted stars. The fits include two components. The black (white) pixels represent excess (depletion) of data relative to the model. The numbers in the top corners of each panel give the highest and lowest values of the residuals, while the percentages on the lower corners give the contribution of different components with corresponding colors.

tion. The contributions of each component are given by the percentages written in the bottom corners. We find that the radially-biased components, if they exist, become less prominent with decreasing metallicity (see Fig. A1 and Fig. B2); similar to pattern in the observations of Belokurov et al. (2018b).

Fig. 3 presents the properties of the higher $\beta$ component of the stellar haloes as inferred by $\mathrm{ED}$, at $9<|z| / \mathrm{kpc}<15$ and $-1<[\mathrm{Fe} / \mathrm{H}]<-0.7$, in the 28 Auriga galaxies. A significant fraction of these galaxies, $\sim 75 \%$, has a halo component with high anisotropy $(\beta>0.7)$. A key point to consider is the component's contribution to the (accreted) stellar halo within the spatial and metallicity selection cuts (see examples in Fig. 2). Recall that the radially-biased component of the MW dominates the mass of the inner stellar halo, as discussed in Sec. 2. Therefore, we identify haloes with high $\beta$ as well as a large fractional contribution to the halo (the top-right corner of Fig. 3) as being similar to the MW. More precisely, we apply the cuts $\beta>0.8$ and contribution $>50 \%$, marked by the dashed lines. Out of 28 Auriga galaxies, 10 $(\sim 1 / 3)$ survive these cuts.

We emphasize that the general conclusions from Fig. 3 do not change when we perform our analysis on all stars, instead of only accreted stars. We indeed tried performing the ED analysis on all stars, and found good fits using three components (1 disk compo- nent and 2 halo-like ones). We find that some of the in-situ stars are potentially associated with the radially-biased components according to their kinematics, and that their formation time is correlated with the merger time of the progenitors of these radially-biased components (more on the progenitors of the radially-biased components in the next section). We plan to address the origin of such in-situ stars in a follow-up paper.

\subsection{Origin of the radially-biased component}

In Fig. 4 we present the dark matter accretion history of the Auriga MW analogues. They are split into two groups, one with a significant radially-biased component (red), and the rest (blue). Solid lines indicate the median mass accreted as a function of time for each group, and the shaded regions correspond to the full range of variation. Even though the sample is small, it is obvious that the galaxies with a significantly radially-biased component grow faster between $t=4-8 \mathrm{Gyr}^{7}$ compared to the rest of the galaxies. A larger sample of galaxies is, however, needed to confirm whether the biased assembly history is statistically significant. The result of

\footnotetext{
$7 t=0$ Gyr corresponds to the big bang.
} 


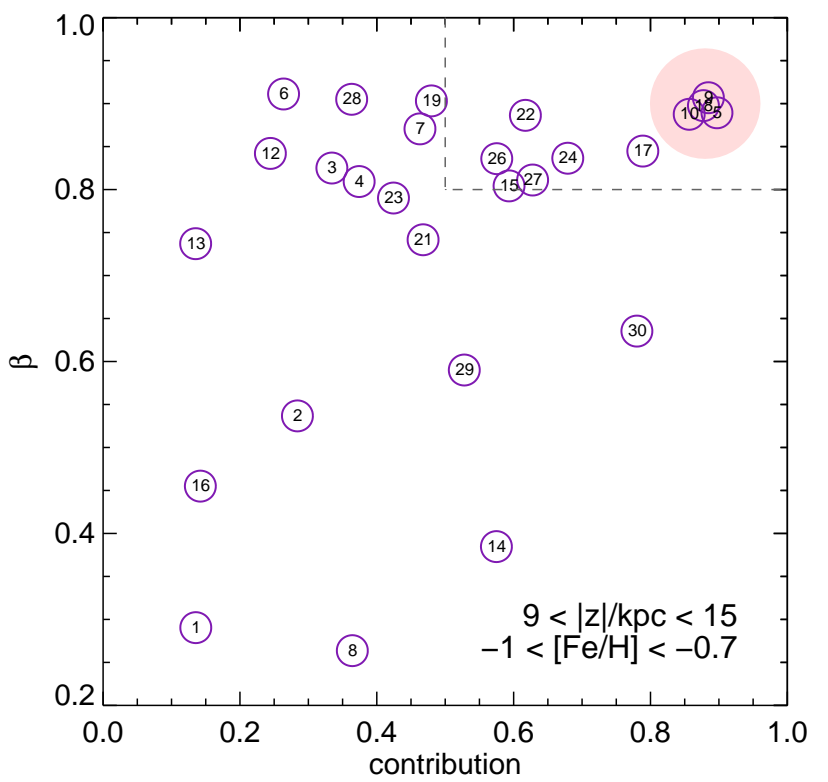

Figure 3. Anisotropy parameter $(\beta)$ of the more radial component of the Auriga stellar haloes, versus their relative contribution to the stellar halo. These results are based on the decomposition of the velocity space (see bottom panels of Fig. 2 and the text for further details). We only consider accreted stars at galactic height of $9<|z| / \mathrm{kpc}<15$ with $-1<[\mathrm{Fe} / \mathrm{H}]<$ -0.7 . Galaxies inside the dashed lines have a dominant, highly radial component similar to the Gaia Sausage. The four haloes highlighted in the topright corner (Au-5, Au-9, Au-10, Au-18) are the extreme cases, also marked in the next plots, where a highly-radial component dominates their inner stellar halo. The small numbers inside each symbol represent the halo number of the Auriga galaxies for reference.

Fig. 4 is broadly consistent with the proposal by Belokurov et al. (2018b) that the origin of the "Gaia Sausage" is a massive, early merger.

We investigate the origin of the prominent radially-biased components (i.e. $\beta>0.8$ and contribution $>50 \%$ ) in the Auriga galaxies by tracking their star particles back in time. We select the (accreted) stars 'associated' with the radially-biased components using: (i) metallicity, $-1.3<[\mathrm{Fe} / \mathrm{H}]<-0.7$; (ii) galactic height, $9<|z| / \mathrm{kpc}<15$; (iii) azimuthal velocity, $\left|V_{\theta}-V_{\text {mean }}\right|<$ $50 \mathrm{~km} \mathrm{~s}^{-1}$; and (iv) radial velocity $V_{\mathrm{r}}>100 \mathrm{~km} \mathrm{~s}^{-1} . V_{\text {mean }}$ refers to the azimuthal velocity of the radially-biased component derived from the ED modeling of the previous section. The radial velocity cut is used to clean the sample from stars potentially associated with a more isotropic component. We checked that the following analysis is not sensitive to the exact values of the aforementioned cuts. The total mass of the sample of stars 'associated' with the radially-biased component is referred to as $M_{\text {radial }}$ in the following analysis.

We identify all of the progenitor galaxies which brought in the stars that ended up in the sample of stars 'associated' with the radially-biased component, in any target galaxy. We then rank the progenitors by their mass contribution $\left(M_{\mathrm{c}}\right)$ to the sample. The progenitor that contributed the most is identified as the main progenitor (or main contributor) of the radially-biased component ${ }^{8}$. Fig. 5

8 According to our definition, the main progenitor is not necessarily the most massive progenitor, but is typically amongst the most massive ones.

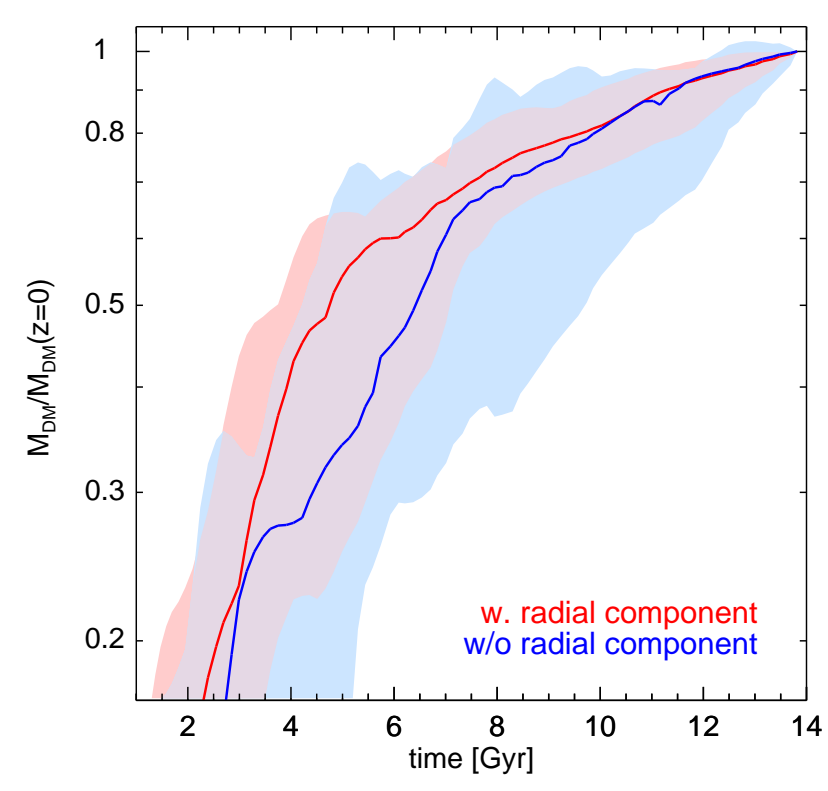

Figure 4. Dark matter accretion history of Auriga galaxies, normalized to the present-day halo virial mass. The red line (shaded region) illustrates the median (full range) of the accretion histories for galaxies with a significant radially-biased stellar halo component (highlighted in the top-right corner of Fig. 3). The blue line and shaded region correspond to the accretion history of the other galaxies.

presents the peak stellar mass of the main progenitor, its relative contribution to the stars associated with the radially-biased component (i.e. $M_{\mathrm{c}} / M_{\text {radial }}$ ), and its merger time with the main progenitors of the MW analogue ${ }^{9}$.

The top panel of Fig. 5 demonstrates that the stars belonging to the radially-biased component predominantly come from a single progenitor: a massive $\left(M_{\text {star }} \sim 10^{9}-10^{10} \mathrm{M}_{\odot}\right)$ galaxy which contributed more than $\sim 50 \%$ to the component. The few cases where the main progenitor contributed less than $\sim 40 \%$, have one or two additional massive progenitors. The merger time of the main contributors, according to the bottom panel of Fig. 5, span the wide range $t \sim 4-8 \mathrm{Gyr}$, with some concentration frequency around $t \sim 4-5$ Gyr. The filled symbols, which correspond to the four cases in the top-right corner of Fig. 3, correspond roughly to a single LMC-like progenitor and a wide range of merger times. The preference for an early merger with a massive dwarf galaxy around $t \sim 4-5$ Gyr explains the behavior of the mass accretion history (illustrated in Fig. 4) of the galaxies with the radially-biased component. The main progenitor of a radially-biased component must have contributed notably to the growth of the MW analogues.

We now examine the contribution of the main progenitor of the radially-biased component to the inner regions of the MW analogues at $z=0$. The top panel of Fig. 6 shows how much of the stellar mass in the inner $20 \mathrm{kpc}$ of the MW analogues were brought in by those progenitors, compared to the total accreted mass within $20 \mathrm{kpc}$. The main progenitors of the radially-biased component contribute a significant fraction to the inner stellar halo, and the contribution is consistently $\sim 1 / 3-1 / 2$ (inside $20 \mathrm{kpc}$ ), except in one case (Au-26). The bottom panel of Fig. 6 shows that the situ-

9 Merger time is defined as the time when Subfind fails to find the merging galaxy as a separate bound substructure. 

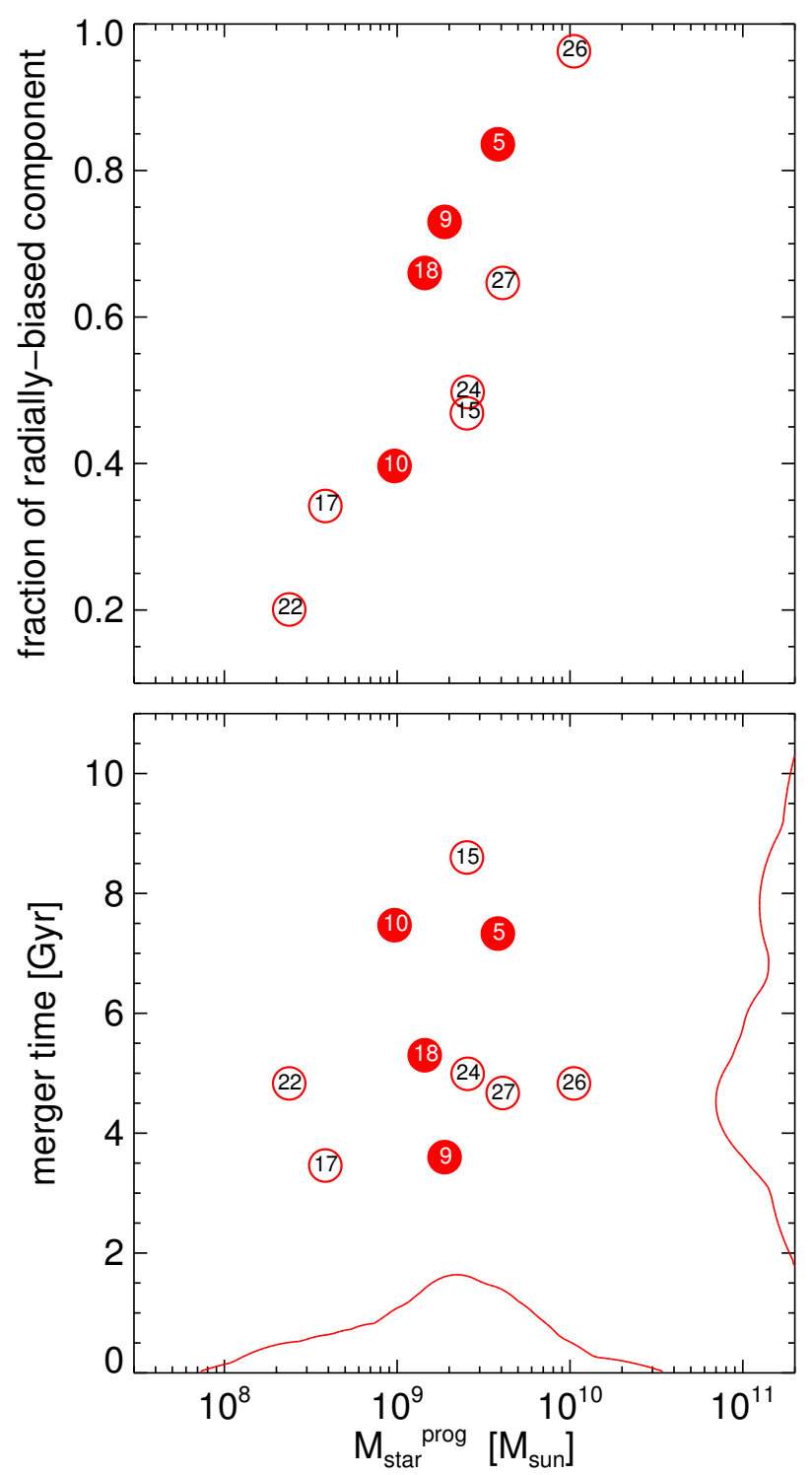

Figure 5. Top: The stellar mass of the progenitor galaxy which contributed the most to the radially-biased component in the Auriga galaxies, versus their mass contribution to that component. See text for full details of the selection of the stars belonging to the radially-biased component. Bottom: Merger time of the main contributors with the [progenitors of the] MW analogues, versus their peak stellar mass. Filled symbols correspond to the four haloes highlighted in the top-right corner of Fig. 3 with extremely anisotropic components dominating the inner halo. The distributions shown on each axis correspond to projecting all of the plotted points on one axis, smoothed with a Epanechnikov kernel.

ation is different for the dark matter (DM): the contribution of the progenitor of the radially-biased stars to the DM in the inner region $(r<20 \mathrm{kpc})$ varies by more than an order of magnitude amongst different haloes, and is much less significant compared to the stellar contribution $(\sim 1-10 \%)$. There are two reasons behind the observed difference between the DM and stellar contributions of the main progenitors. First, stars are more centrally concentrated than the DM; hence they occupy different regions of the energy-angular momentum space after becoming tidally stripped. The progenitors start to lose their DM before their stars, and therefore deposit their
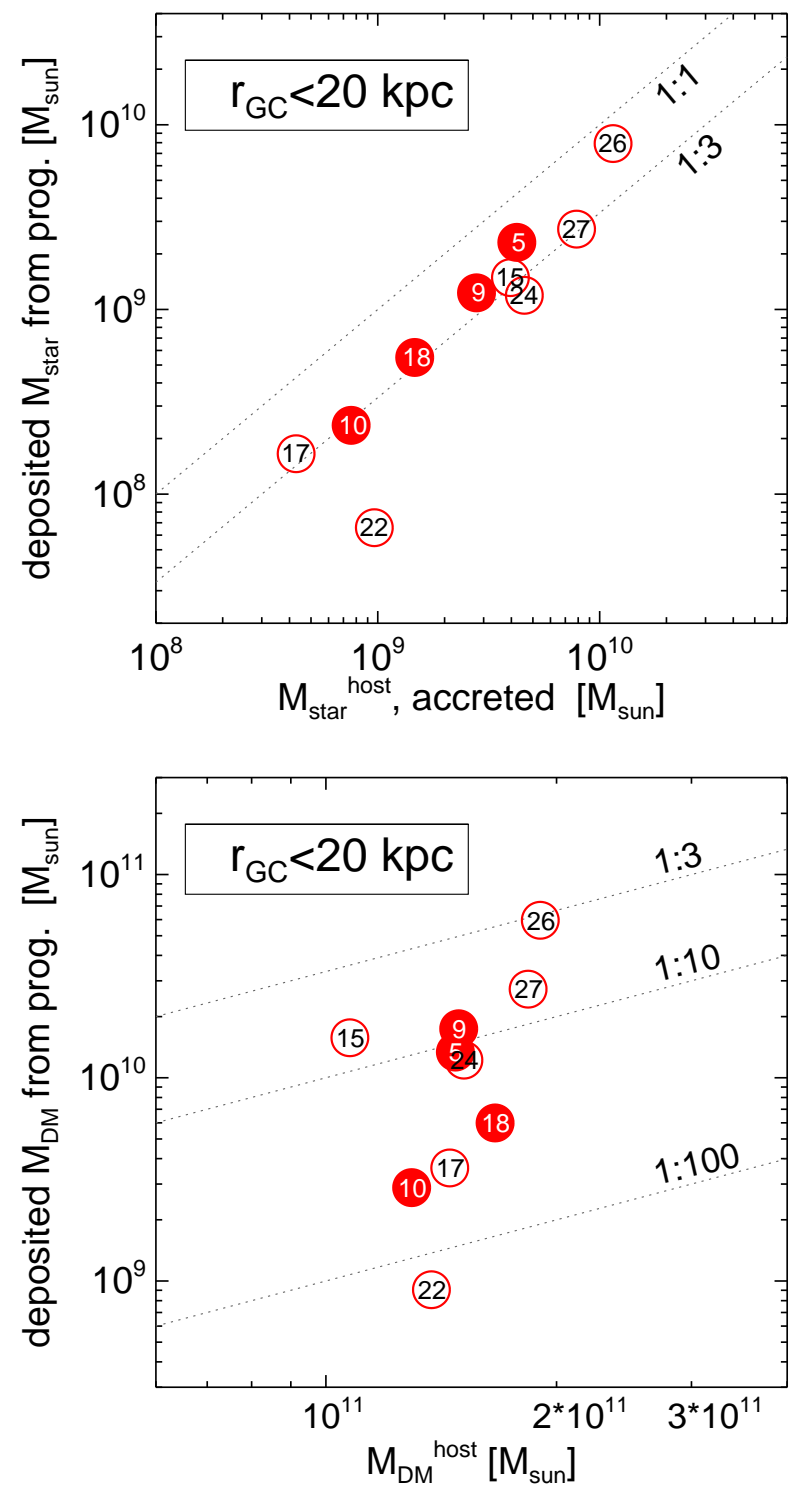

Figure 6. Top: Contribution of the main progenitor of the radially-biased component to the stellar mass in the inner $20 \mathrm{kpc}$ of the Auriga galaxies at $z=0$, versus the total accreted stellar mass in the same volume. The diagonal lines correspond to the $1: 1$ and 1:3 mass ratios as indicated by the labels. Bottom: DM contribution of the main progenitor to the inner $20 \mathrm{kpc}$, versus the total DM mass in $r<20 \mathrm{kpc}$.

DM in a larger volume. Second, the stellar mass-halo mass relation at the faint end is so steep in $\Lambda$ CDM that almost all dwarf galaxies live in similar haloes of mass $\sim 10^{10} \mathrm{M}_{\odot}$ (Eke et al. 2004; Behroozi et al. 2013; Sawala et al. 2016; Deason et al. 2016; Fattahi et al. 2018; Simpson et al. 2018). As a consequence, while the build up of the accreted stellar halo can be dominated by the merger of a few relatively bright dwarf galaxies, their DM contribution is more representative of fainter dwarfs (see, Wang et al. 2011, for a detail analysis on the build-up of MW-like haloes). 


\section{SUMMARY AND DISCUSSION}

We use the Auriga magneto-hydrodynamical simulations of MWlike galaxies to study the origin and frequency of a highly radial ( $\beta \sim 0.9$ ) and metal-rich component of the inner halo, recently reported by Belokurov et al. (2018b) using Gaia DR1. This component is believed to be the same population discussed by Helmi et al. (2018). Firstly, we present an updated version of the results of Belokurov et al. (2018b) using Gaia DR2. We confirm the existence of a large group of stars on highly radial orbits with $\beta \sim 0.86$ (the "Gaia Sausage"), in Gaia DR2.

Secondly, we search for the phase-space signatures of such a radially-biased population of star particles amongst Auriga galaxies and find that they are relatively common: around $1 / 3$ of the simulated galaxies have a significant population of stars residing above and below the disc, with $[\mathrm{Fe} / \mathrm{H}] \sim-1$, which resemble the observations. In addition, we find that the significance of the sausage-like components, when they exist, always decreases at lower $[\mathrm{Fe} / \mathrm{H}]$. This metallicity dependence trend has also been observed in the MW by Belokurov et al. (2018b). Essentially, the phase space of all galaxies looks similar at metallicities below $[\mathrm{Fe} / \mathrm{H}] \sim-2.0$ and is dominated by a halo component with $\beta \sim 0.3$.

We demonstrate that the accretion history of Auriga galaxies with a radially-biased component is biased, on average, towards steeper growth at $t \sim 4-6 \mathrm{Gyr}$, suggesting more mergers at those early times. It is intriguing that Mackereth et al. (2018) find, using the EAGLE simulations, a similar early accretion bias for galaxies that show alpha-bimodality in their stars. This is supported also by the study of Grand et al. (2018), which found that the early accretion of a gas-rich major merger at around redshift 2 is required to form a prominent alpha-bimodality. The connection between alphabimodality and the radially-biased components is worth pursuing in follow-up studies.

By tracing back the star particles associated with the radiallybiased component of each Auriga galaxy, we find that their main progenitor is typically a massive dwarf galaxy with peak stellar mass of $10^{9}-10^{10} \mathrm{M}_{\odot}$, which merged with the progenitor of the MW analogues $\sim 6-10$ Gyr ago. Such a massive dwarf progenitor also explains why the radially-biased component is prominent at relatively higher metallicities $([\mathrm{Fe} / \mathrm{H}] \sim-1)$ : it is a consequence of the tight stellar mass-metallicity relation (Kirby et al. 2013).

Given that the main progenitors of the radially-biased components in Auriga halos are as massive as the LMC (or even more massive), their merger with the MW progenitor can have a large impact on the structure of the disc. In some cases the merger almost completely destroys the disc, and a new younger disc has to regrow. We note that these mergers are different from the those reported by Gómez et al. (2017) in the Auriga galaxies which brought in exsitu disc stars. These have different orbits which deposit the tidally stripped stars in circular orbits co planar with the disc, as opposed to the radially-biased components studies here. We will present a thorough analysis of the mergers which made the "Gaia Sausage"like components, and their influence on the evolution of the MW, in a subsequent paper.

Finally, we find that the star particles brought in by the main progenitors of the radially-biased components make up $\sim 30 \%-$ $50 \%$ of the total amount of the accreted stars in the inner $20 \mathrm{kpc}$ of the MW analogues at $z=0$. The dark matter contribution of the progenitors to the same volume, however, is much lower and varies significantly between different haloes $(\sim 1 \%-10 \%)$. This is a consequence of the inefficient galaxy formation at low mass scales, as well as the difference in the tidal stripping of dark matter particles and stars from the progenitor dwarf. Thus the accreted stellar halo does not directly trace the build-up of the DM halo.

\section{ACKNOWLEDGEMENTS}

We are grateful to the referee, J. Mackereth, for his helpful comments which improved our paper. AF is supported by a European Union COFUND/Durham Junior Research Fellowship (under EU grant agreement no. 609412). T AD is supported by a Royal Society University Research Fellowship. AF, AD and CSF also acknowledge the support from the STFC grant ST/P000541/1. FAG acknowledges financial support from FONDECYT Regular 1181264 , and funding from the Max Planck Society through a Partner Group grant. RG acknowledges support by the DFG Research Centre SFB-881 'The Milky Way System' through project A1. CSF acknowledges support from the European Research Council (ERC) through Advanced Investigator Grant DMIDAS (GA 786910). The research leading to these results has received funding from the European Research Council under the European Union's Seventh Framework Programme (FP/2007-2013) / ERC Grant Agreement n. 308024.

This work used the DiRAC Data Centric system at Durham University, operated by ICC on behalf of the STFC DiRAC HPC Facility (www.dirac.ac.uk). This equipment was funded by BIS National E-infrastructure capital grant ST/K00042X/1, STFC capital grant ST/H008519/1, and STFC DiRAC Operations grant ST/K003267/1 and Durham University. DiRAC is part of the National E-Infrastructure.

\section{REFERENCES}

Ahn C. P., et al., 2012, ApJS, 203, 21

Amorisco N. C., 2017, MNRAS, 464, 2882

Behroozi P. S., Marchesini D., Wechsler R. H., Muzzin A., Papovich C., Stefanon M., 2013, ApJ, 777, L10

Belokurov V., et al., 2006, ApJ, 642, L137

Belokurov V., Deason A. J., Koposov S. E., Catelan M., Erkal D., Drake A. J., Evans N. W., 2018a, MNRAS, 477, 1472

Belokurov V., Erkal D., Evans N. W., Koposov S. E., Deason A. J., 2018b, MNRAS, 478, 611

Bergemann M., et al., 2018, Nature, 555, 334

Bird S. A., Xue X.-X., Liu C., Shen J., Flynn C., Yang C., 2018, preprint, (arXiv: 1805.04503)

Bonaca A., Conroy C., Wetzel A., Hopkins P. F., Kereš D., 2017, ApJ, 845, 101

Bovy J., Hogg D. W., Roweis S. T., 2011, Annals of Applied Statistics, 5

Brook C. B., Kawata D., Gibson B. K., Flynn C., 2003, ApJ, 585, L125

Brook C. B., Di Cintio A., Knebe A., Gottlöber S., Hoffman Y., Yepes G., Garrison-Kimmel S., 2014, ApJ, 784, L14

Buist H. J. T., Helmi A., 2015, A\&A, 584, A120

Bullock J. S., Johnston K. V., 2005, ApJ, 635, 931

Carollo D., et al., 2007, Nature, 450, 1020

Carollo D., et al., 2010, ApJ, 712, 692

Chiba M., Beers T. C., 2001, ApJ, 549, 325

Coşkunoğlu B., et al., 2011, MNRAS, 412, 1237

Cooper A. P., et al., 2010, MNRAS, 406, 744

Cooper A. P., Parry O. H., Lowing B., Cole S., Frenk C., 2015, MNRAS, 454,3185

Crain R. A., et al., 2015, MNRAS, 450, 1937

Cropper M., et al., 2018, preprint, (arXiv:1804.09369)

Davis M., Efstathiou G., Frenk C. S., White S. D. M., 1985, ApJ, 292, 371

De Lucia G., Helmi A., 2008, MNRAS, 391, 14

Deason A. J., Belokurov V., Evans N. W., 2011, MNRAS, 416, 2903 
Deason A. J., Belokurov V., Evans N. W., Johnston K. V., 2013, ApJ, 763, 113

Deason A. J., Belokurov V., Weisz D. R., 2015, MNRAS, 448, L77

Deason A. J., Mao Y.-Y., Wechsler R. H., 2016, ApJ, 821, 5

Deason A. J., Belokurov V., Koposov S. E., Gómez F. A., Grand R. J., Marinacci F., Pakmor R., 2017, MNRAS, 470, 1259

Deason A. J., Belokurov V., Koposov S. E., 2018a, MNRAS, 473, 2428

Deason A. J., Belokurov V., Koposov S. E., Lancaster L., 2018b, ApJ, 862, L1

Eke V. R., et al., 2004, MNRAS, 355, 769

Erkal D., Sanders J. L., Belokurov V., 2016, MNRAS, 461, 1590

Fattahi A., Navarro J. F., Frenk C. S., Oman K. A., Sawala T., Schaller M., 2018, MNRAS, 476, 3816

Fernández-Alvar E., Tissera P. B., Carigi L., Schuster W. J., Beers T. C., Belokurov V. A., 2018, preprint, (arXiv:1809.02368)

Frenk C. S., Evrard A. E., White S. D. M., Summers F. J., 1996, ApJ, 472, 460

Gaia Collaboration et al., 2016, A\&A, 595, A1

Gaia Collaboration Brown A. G. A., Vallenari A., Prusti T., de Bruijne J. H. J., Babusiaux C., Bailer-Jones C. A. L., 2018, preprint, (arXiv: 1804.09365)

Garrison-Kimmel S., Boylan-Kolchin M., Bullock J. S., Lee K., 2014, MNRAS, 438, 2578

Gómez F. A., Helmi A., 2010, MNRAS, 401, 2285

Gómez F. A., Minchev I., O’Shea B. W., Beers T. C., Bullock J. S., Purcell C. W., 2013, MNRAS, 429, 159

Gómez F. A., et al., 2017, MNRAS, 472, 3722

Grand R. J. J., et al., 2017, MNRAS, 467, 179

Grand R. J. J., et al., 2018, MNRAS, 474, 3629

Hayes C. R., et al., 2018a, ApJ, 852, 49

Hayes C. R., et al., 2018b, ApJ, 859, L8

Haywood M., Di Matteo P., Lehnert M. D., Snaith O., Khoperskov S., Gómez A., 2018, ApJ, 863, 113

Helmi A., Cooper A. P., White S. D. M., Cole S., Frenk C. S., Navarro J. F., 2011, ApJ, 733, L7

Helmi A., Babusiaux C., Koppelman H. H., Massari D., Veljanoski J., Brown A. G. A., 2018, preprint, (arXiv:1806.06038)

Ibata R. A., Gilmore G., Irwin M. J., 1994, Nature, 370, 194

Ibata R., Irwin M., Lewis G. F., Stolte A., 2001, ApJ, 547, L133

Iorio G., Belokurov V., 2018, preprint, (arXiv: 1808.04370 )

Jenkins A., 2013, MNRAS, 434, 2094

Jethwa P., Erkal D., Belokurov V., 2018, MNRAS, 473, 2060

Johnston K. V., Bullock J. S., Sharma S., Font A., Robertson B. E., Leitner S. N., 2008, ApJ, 689, 936

Katz D., et al., 2018, preprint, (arXiv:1804.09372)

Kirby E. N., Cohen J. G., Guhathakurta P., Cheng L., Bullock J. S., Gallazzi A., 2013, ApJ, 779, 102

Koposov S. E., et al., 2012, ApJ, 750, 80

Kruijssen J. M. D., Pfeffer J. L., Reina-Campos M., Crain R. A., Bastian N., 2018, MNRAS,

Lancaster L., Belokurov V., Evans N. W., 2018a, preprint, (arXiv:1804.09181)

Lancaster L., Koposov S. E., Belokurov V., Evans N. W., Deason A. J., 2018b, preprint, (arXiv:1807.04290)

Laporte C. F. P., Johnston K. V., Gómez F. A., Garavito-Camargo N., Besla G., 2018, MNRAS,

Mackereth J. T., et al., 2018, preprint, (arXiv:1808.00968)

Majewski S. R., Skrutskie M. F., Weinberg M. D., Ostheimer J. C., 2003, ApJ, 599, 1082

McCarthy I. G., Font A. S., Crain R. A., Deason A. J., Schaye J., Theuns T., 2012, MNRAS, 420, 2245

Meza A., Navarro J. F., Abadi M. G., Steinmetz M., 2005, MNRAS, 359, 93

Monachesi A., et al., 2018, preprint, (arXiv: 1804.07798 )

Moster B. P., Naab T., White S. D. M., 2013, MNRAS, 428, 3121

Myeong G. C., Evans N. W., Belokurov V., Sanders J. L., Koposov S. E., 2018a, preprint, (arXiv:1805.00453)
Myeong G. C., Evans N. W., Belokurov V., Sanders J. L., Koposov S. E., 2018b, ApJ, 856, L26

Navarro J. F., Abadi M. G., Venn K. A., Freeman K. C., Anguiano B., 2011, MNRAS, 412, 1203

Necib L., Lisanti M., Belokurov V., 2018, preprint, (arXiv: 1807.02519)

Newberg H. J., et al., 2003, ApJ, 596, L191

Nissen P. E., Schuster W. J., 2010, A\&A, 511, L10

Peñarrubia J., Benson A. J., Martínez-Delgado D., Rix H. W., 2006, ApJ, 645,240

Planck Collaboration et al., 2014, A\&A, 571, A16

Price-Whelan A. M., Johnston K. V., Sheffield A. A., Laporte C. F. P., Sesar B., 2015, MNRAS, 452, 676

Purcell C. W., Bullock J. S., Tollerud E. J., Rocha M., Chakrabarti S., 2011, Nature, 477, 301

Read J. I., Iorio G., Agertz O., Fraternali F., 2017, MNRAS, 467, 2019

Rodriguez-Gomez V., et al., 2016, MNRAS, 458, 2371

Sartoretti P., et al., 2018, preprint, (arXiv: 1804.09371 )

Sawala T., et al., 2016, MNRAS, 456, 85

Scannapieco C., White S. D. M., Springel V., Tissera P. B., 2009, MNRAS, 396, 696

Schaye J., et al., 2015, MNRAS, 446, 521

Schönrich R., 2012, MNRAS, 427, 274

Sesar B., Jurić M., Ivezić Ž., 2011, ApJ, 731, 4

Simion I. T., Belokurov V., Koposov S. E., 2018, preprint, (arXiv:1807.01335)

Simpson C. M., Grand R. J. J., Gómez F. A., Marinacci F., Pakmor R., Springel V., Campbell D. J. R., Frenk C. S., 2018, MNRAS, 478, 548

Springel V., 2010, MNRAS, 401, 791

Springel V., Yoshida N., White S. D. M., 2001, New Astronomy, 6, 79

Springel V., et al., 2008, MNRAS, 391, 1685

Tissera P. B., Scannapieco C., Beers T. C., Carollo D., 2013, MNRAS, 432, 3391

Tolstoy E., Hill V., Tosi M., 2009, ARA\&A, 47, 371

Venn K. A., Irwin M., Shetrone M. D., Tout C. A., Hill V., Tolstoy E., 2004, AJ, 128, 1177

Wang J., et al., 2011, MNRAS, 413, 1373

Watkins L. L., et al., 2009, MNRAS, 398, 1757

Wegg C., Gerhard O., Bieth M., 2018, preprint, (arXiv:1806.09635)

Xue X.-X., et al., 2011, ApJ, 738, 79

Yanny B., et al., 2009, ApJ, 700, 1282

Zolotov A., Willman B., Brooks A. M., Governato F., Brook C. B., Hogg D. W., Quinn T., Stinson G., 2009, ApJ, 702, 1058

de Boer T. J. L., Belokurov V., Beers T. C., Lee Y. S., 2014, MNRAS, 443, 658

de Boer T. J. L., Belokurov V., Koposov S. E., 2018, MNRAS, 473, 647

\section{APPENDIX A: PHASE-SPACE EXAMPLES OF AURIGA GALAXIES}

Here we present the 2D velocity space of accreted stars (azimuthal vs radial components), on a full grid of height and metallicity, for 2 examples of Auriga galaxies: one where the "Gaia sausage"component is prominent (Fig. A1) and one with no notable highly radial component (Fig. A2). The galactic height increases from the top row to bottom in the range $|z|=1-15 \mathrm{kpc} ;[\mathrm{Fe} / \mathrm{H}]$ increases from -4 to -0.7 , from left to right.

The component at higher metallicities with $v_{r} \sim 0 \mathrm{~km} \mathrm{~s}^{-1}$ and $v_{\theta} \sim 200 \mathrm{~km} \mathrm{~s}^{-1}$ is the disc of the galaxies. In Fig. A1, the highly radial component is obvious at higher metallicities, and because less and less obvious at lower metallicities. We also note that the radial component is more visible at higher $|z|$; this is due to the fact that the disc is more dominant at lower $|z|$ over the halo component. Therefore, the difference due to the existence (or lack) 


\section{A. Fattahi et al.}

of the highly radial component is apparent at higher $|z|$ and higher $[\mathrm{Fe} / \mathrm{H}]$ (bottom-right panels).

\section{APPENDIX B: DECOMPOSITION OF PHASE-SPACE}

We adopt the following, 2step procedure to decompose the phasespace of stars in each Auriga galaxy.

(i) We start with the $3 \mathrm{D}$ velocity space of stars with $[\mathrm{Fe} / \mathrm{H}]<$ -0.7 , at different heights. This is equivalent to stacking columns of Fig. A1. We decompose the velocity space at each height, using the "extreme deconvolution" method of Bovy et al. (2011), into three components (velocity ellipsoids) with free parameters. The initialization of parameters ensures that the three components include a disc and two halo-like components. Fig. B1 illustrates this step.

(ii) In the next step, we divide each height into different metallicity bins (equivalent of Fig. A1). After fixing the centre and shape of the velocity ellipsoids from the previous step, we fit them to different metallicities and let the amplitude (contribution) vary. Fig. B2 shows the residuals of the fit to an example Auriga galaxy. The rows and columns are similar to Figs. A1 and A2. Note that the the velocity ellipses (the models) are fixed across any given row by our procedure.

This paper has been typeset from a $\mathrm{T}_{\mathrm{E}} \mathrm{X} / \mathrm{L} \mathrm{T} \mathrm{E} X$ file prepared by the author. 

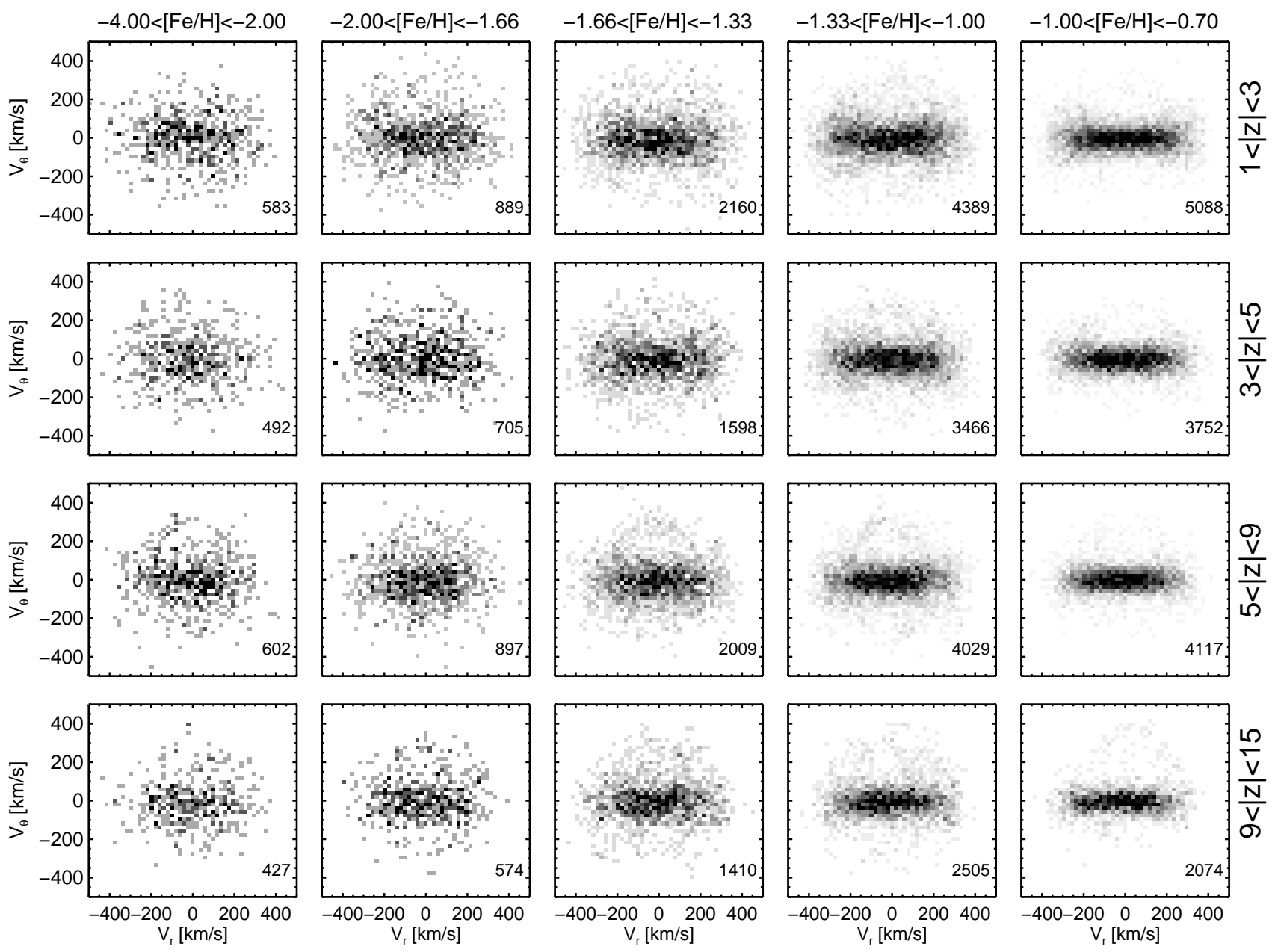

Figure A1. 2D velocity space (azimuthal vs radial) of accreted stars for an Auriga halo (Au-5) at different heights $|z|$ and [Fe/H]. Rows from top to bottom correspond to as $1-3,3-5,5-9,9-15 \mathrm{kpc}$ above and below the galactic disc. Different columns represent different $[\mathrm{Fe} / \mathrm{H}]$ of $<-2,-2.0,-1.7$, $-1.7,-1.3,-1.3,-1.0$, and $-1.0,-0.7$. This is a clear example of a galaxy with a highly radial population of stars at higher [Fe/H], similar to the Gaia observations of the MW (Belokurov et al. 2018b). 


\section{A. Fattahi et al.}
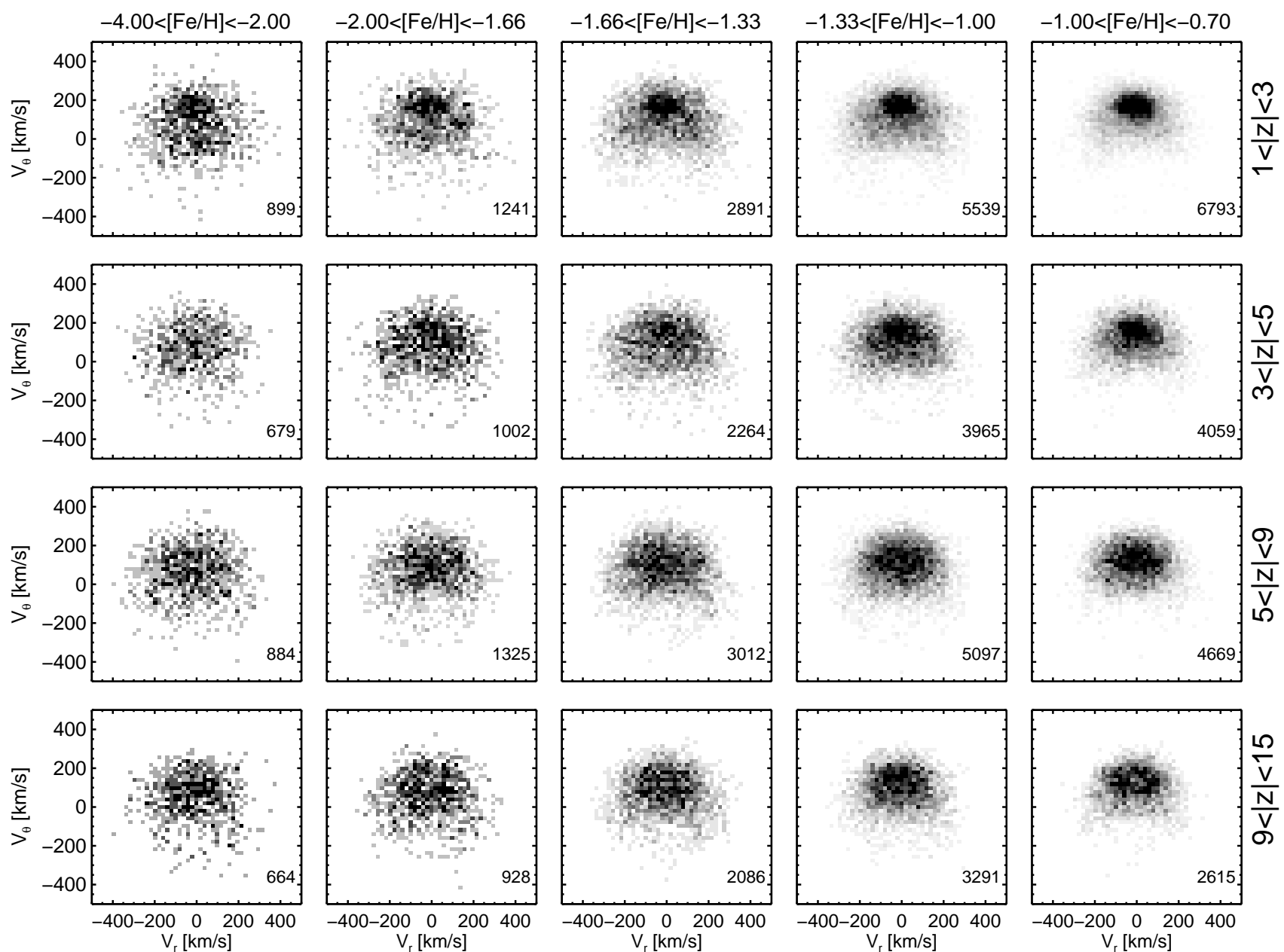

Figure A2. Similar to Fig. A1, but for a galaxy (Au-16) with no considerable radially-biased ("sausage"-like) component. 

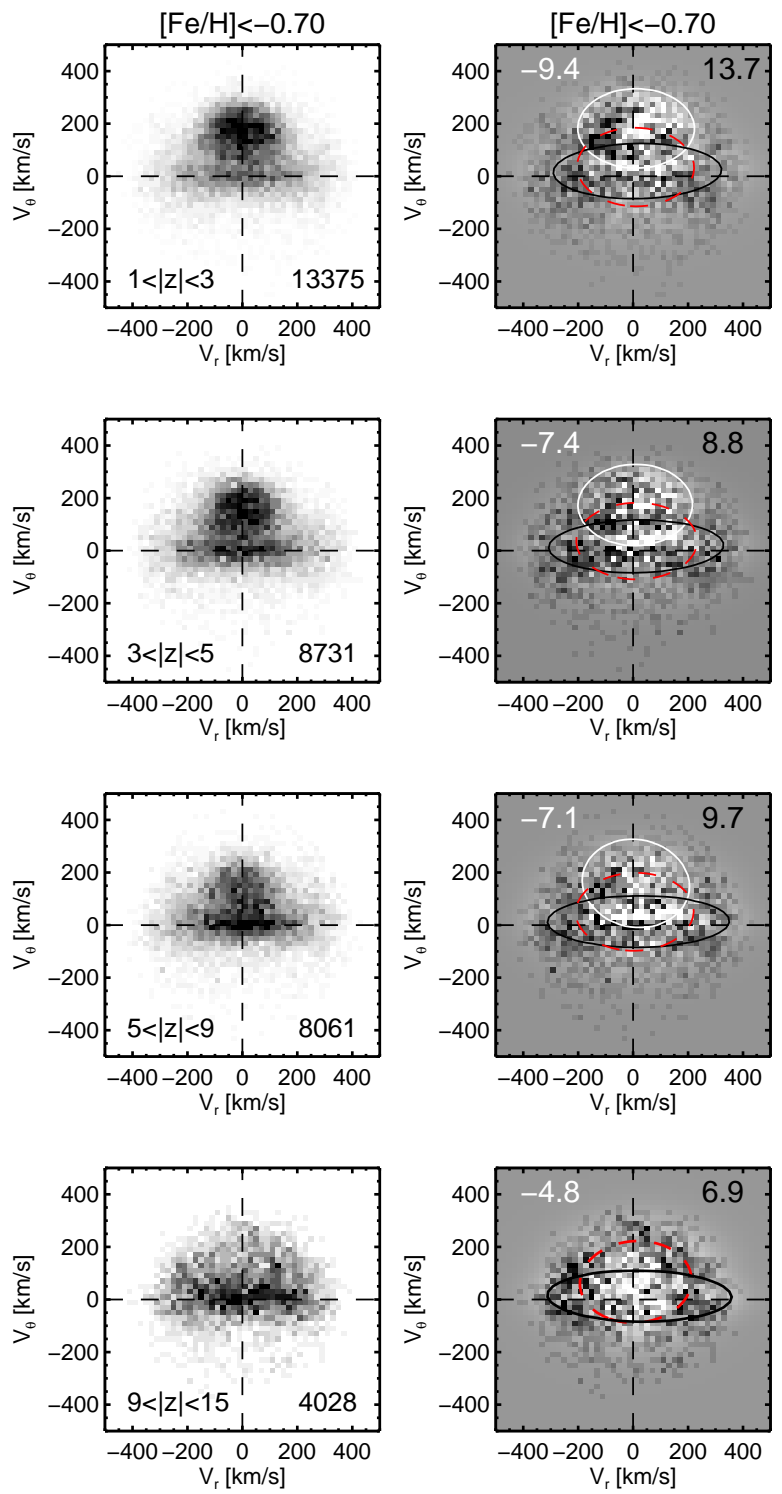

Figure B1. An illustration of the first step of the fitting procedure: the deconvolution of the velocity space into two or three Gaussian components, after combining $[\mathrm{Fe} / \mathrm{H}]$ bins at each galactic height. The left column shows the velocity space at different heights (different rows), while the right column shows the residuals of the corresponding model. The three components of the model at each height include three velocity ellipsoids; one for the disc (white ellipse) and two for the halo-like components (black and red ellipses). The two component fit (last row) include only two halo-like components. The example shown is Au-6. 

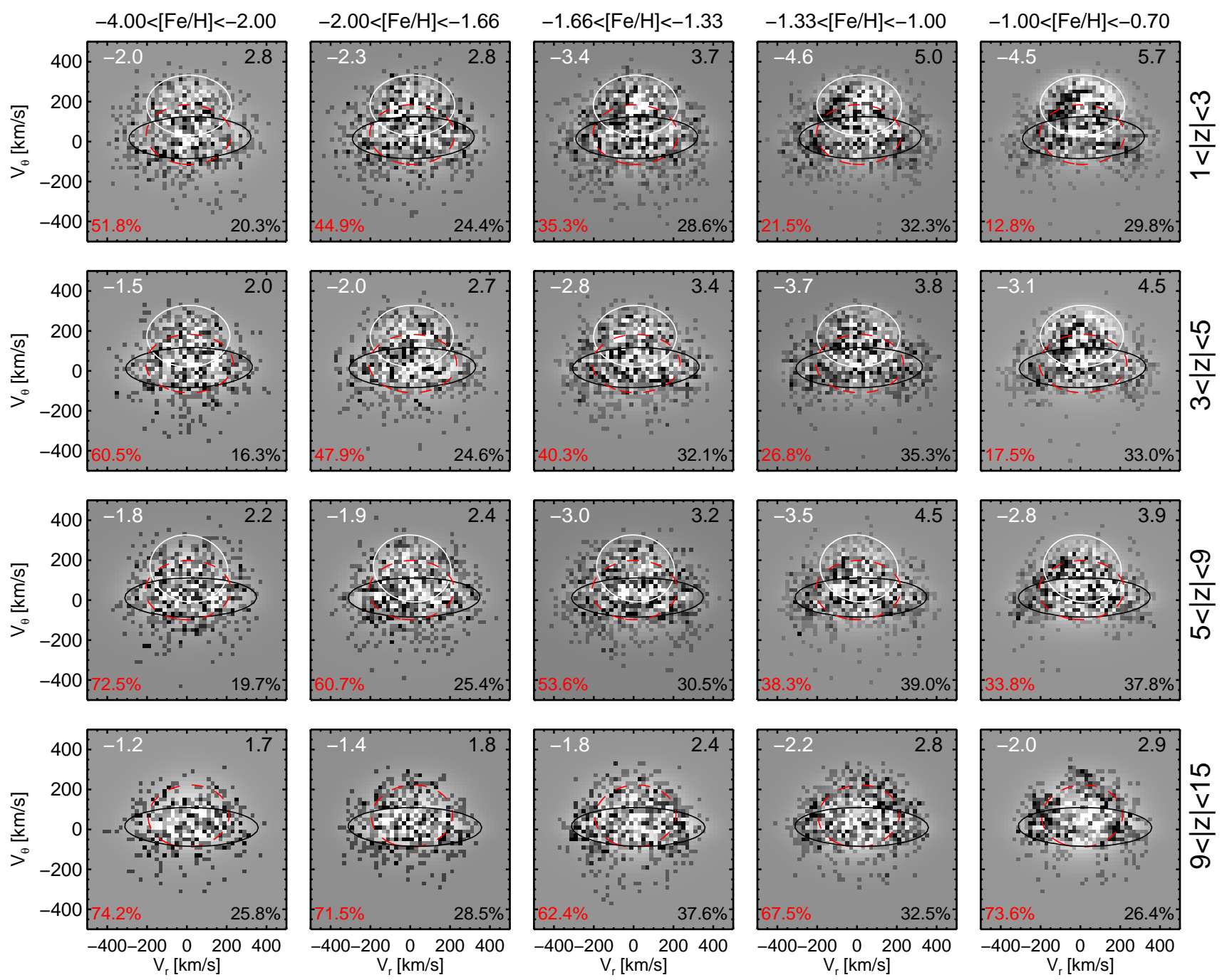

Figure B2. Second step of the fitting procedure. The centre and shape of the velocity ellipsoids from the previous step (Fig. B1) are fixed, and the amplitudes of the ellipsoids vary across different $[\mathrm{Fe} / \mathrm{H}]$ bins. The residuals are shown here in a grid of height (rows) and metallicity (columns). The numbers in the top corners of each panel show the highest and lowest values of residuals, while the percentages in the lower corners show the contribution of different components with corresponding colors. This example is the same galaxy as Fig. B1 